\title{
BMJ Open Protocol for a randomised controlled trial of interventions to promote adoption and maintenance of physical activity in adults with mental illness
}

Justin J Chapman, ${ }^{1,2,3}$ Shuichi Suetani, ${ }^{2,4,5,6}$ Dan Siskind, ${ }^{2,5,4}$ Steve Kisely, ${ }^{2,5,7}$ Michael Breakspear, ${ }^{1,8}$ Jacqueline H Byrne, ${ }^{1,8}$ Sue Patterson ${ }^{8,9}$

To cite: Chapman JJ, Suetani S, Siskind D, et al. Protocol for a randomised controlled trial of interventions to promote adoption and maintenance of physical activity in adults with mental illness. BMJ Open 2018;8:e023460. doi:10.1136/ bmjopen-2018-023460

- Prepublication history for this paper is available online. To view these files, please visit the journal online (http://dx.doi. org/10.1136/bmjopen-2018023460).

Received 7 April 2018 Revised 11 June 2018 Accepted 21 June 2018

Check for updates

(C) Author(s) (or their employer(s)) 2018. Re-use permitted under CC BY-NC. No commercial re-use. See rights and permissions. Published by BMJ.

For numbered affiliations see end of article.

Correspondence to

Dr. Justin J Chapman;

JustinJChapman@gmail.com

\section{ABSTRACT}

Introduction Physical activity (PA) has diverse benefits for physical and mental health and can reduce symptoms of mental illness. Adults with mental illness face practical, psychosocial and socioeconomic barriers to adopting and maintaining $\mathrm{PA}$, and it is unclear how to effectively promote PA in this group. Supervised exercise interventions provide high support but may not promote autonomous motivation, which is important for PA maintenance. The aim of this study is to compare the effectiveness of two interventions to promote PA in adults with mental illness.

Methods and analysis This is a randomised controlled trial of two interventions to promote PA: (1) supervised exercise and gym membership and (2) motivational discussions and self-monitoring of PA using fitness trackers. The intervention duration is 16 weeks, including 8 weeks of weekly supervised group sessions, and 8 weeks of access to the gym or fitness tracker unsupervised. Participants are community-dwelling adults recruited from outpatient clinics of public mental health services. The primary outcome is PA adoption assessed using GENEActiv accelerometers worn continuously over 8 weeks. Secondary outcomes measured at baseline, postintervention (8 weeks) and follow-up (16 weeks), include exercise motivation, psychological distress and self-reported PA assessed using self-administered questionnaires and indicators of physical health measured by a researcher blinded to allocation (blood pressure, weight, waist circumference, 6 min walk test). Participant experiences will be assessed using qualitative focus groups with analysis informed by a theoretical model of behaviour (COM-B).

Ethics and dissemination Ethics approval has been obtained from the Royal Brisbane and Women's Hospital (HREC/17/QRBW/302). We plan to submit a manuscript on protocol development from pilot work, and a manuscript of the results to a peer-reviewed journal. Results will be presented at conferences, community and consumer forums and hospital grand rounds.

Trial registration number ACTRN12617001017314; Preresults.
Strength and limitations of this study

- This study uses a robust methodological design, and the interventions are based on theoretical model of behaviour to enhance interpretation and generalisability of findings.

- An objective measure of physical activity (PA) is used continuously during the intervention to allow reliable assessment of PA behaviour change.

- Recruitment will be non-probabilistic, so the resulting cohort may not be a representative sample of adults receiving public mental health services.

- Use of a self-report measure of PA during the follow-up period limits assessment of PA maintenance.

\section{INTRODUCTION}

The benefits of physical activity (PA) for mental and physical health are widely recognised. ${ }^{1} \mathrm{PA}$ and exercise (PA to enhance or maintain fitness) can improve metabolic risk factors, protect against chronic physical conditions such as cardiovascular disease and improve psychosocial well-being and longevity. ${ }^{2}$ The WHO PA guidelines are to accumulate 150-300 min per week of moderate-to-vigorous activity. ${ }^{4}$ About a third of the population globally do not meet these guidelines, with inactivity more prevalent in high-income countries. ${ }^{5}$ Policy initiatives and public health campaigns to increase PA can be effective $^{6}$; however, research has highlighted the importance of targeting campaigns to specific subgroups, ${ }^{7}$ and people experiencing socioeconomic and psychosocial barriers may need more focused support to adopt and maintain an active lifestyle. ${ }^{8}$

One such population group is adults with mental illness. This group are at higher risk of developing cardiovascular and metabolic conditions, ${ }^{9}$ and have lower levels of $\mathrm{PA}^{10}$ than the general population. In addition to the acknowledged physical and psychosocial 
benefits of PA, PA can reduce symptoms of depression, ${ }^{11}$ anxiety ${ }^{12}$ and schizophrenia, ${ }^{13}$ and improve quality of life in adults with mental illness. ${ }^{14}$ Adults with mental illness face complex barriers to adopting and maintaining an active lifestyle: social isolation, medication side effects and illness symptoms are among many factors that have been described as hindering PA behaviour change. ${ }^{15} \mathrm{PA}$ intervention studies have demonstrated feasibility ${ }^{16}$ and are associated with improved health outcomes ${ }^{14}$; however, little is known about how effective such interventions are at influencing PA outside supervised sessions, which is important for maintaining health benefits. ${ }^{17}$ Given the potential for PA to improve health and well-being in this group, the implementation of interventions to promote PA in mental health services is increasingly advocated. ${ }^{18} 19$

Research has highlighted the importance of autonomous motivation in adoption and maintenance of PA for adults with mental illness. ${ }^{20}$ Cross-sectional studies indicate that the motivational mechanisms that influence PA behaviour in adults with mental illness are not dissimilar to the general population, and are independent of psychiatric diagnosis and medication use, ${ }^{21}$ indicating that established behaviour change theories are generalisable to this group. However, the importance of tailoring interventions to focus on the specific barriers and facilitators experienced by adults with mental illness has been emphasised. ${ }^{8}$ The few studies of interventions to improve PA motivation in adults with mental illness have not based their evaluation on a theoretical model of behaviour, which is critical for understanding potential mechanisms of behaviour change, or have not related motivational outcomes to PA behaviour to examine potential causal relationships. ${ }^{22}$ There is a need for theory-based empirical evidence on how to positively impact PA motivation and behaviour in adults with mental illness.

The Behaviour Change Wheel (BCW) framework is an overarching model of behaviour, which can be used to design and evaluate behaviour change interventions with the view of improving adherence and effectiveness. ${ }^{23}$ The BCW identifies nine 'intervention functions' that potentially influence any given target behaviour (Box 1, footnote $(*)$ ), and explains behaviour change through the COM-B model, in which capability $(\mathrm{C})$, opportunity $(\mathrm{O})$ and motivation $(\mathrm{M})$ interact to generate behaviour (B). ${ }^{23}$ Motivation and Capability are facets of the individual (physical and psychological capabilities; reflective and automatic motivations), and Opportunity encompasses factors outside the individual that prompt or enable the behaviour. Evaluating and comparing different interventions using this theoretical behavioural framework will provide valuable insight into effectiveness and mechanisms of action of interventions designed to promote PA in adults with mental illness.

The range of interventions that might promote PA is diverse, in that different combinations of intervention functions can be used to impact capability, opportunity and motivation. Trials designed to assess the efficacy of exercise on health outcomes for adults with mental illness
Box 1 Description of intervention functions* and behaviour change techniquest used in the interventions

\section{Gym exercise intervention}

\section{Capability}

Physical capability

- Training: verbal instruction (BCT21) and demonstration (BCT22) of different exercises by the accredited exercise physiologist (AEP). Participants then complete the exercises with technique correction from the AEP (not coded).

\section{Opportunity}

\section{Physical opportunity}

- Enablement. provision of access to exercise facility (not coded) and providing information on where and when to exercise (ie, at the gym during opening times for supervised and unsupervised sessions) (BCT20).

Social opportunity

- Environmental restructuring: exercise sessions delivered in groups. Motivation

Reflective

- Education about purpose and general health consequences of specific exercises (BCT1), eg, to improve posture, core stability, interval training. Automatic

- Environmental restructuring: self-monitoring of exercises completed using a diary for supervised and unsupervised sessions (BCT16).

\section{Motivational intervention \\ Capability \\ Psychological capability}

- Training: behavioural goal setting (BCT5) and setting graded weekly tasks (BCT9) in week 1. Reviewing progress and reassessing goals (BCT10) each week. Refining goals in subsequent weeks, by: identifying preferred available community opportunities (eg, activity groups; walking routes, etc) (BCT20) and action planning for physical activity (PA) in contexts of commuting, leisure, occupational and incidental activity (BCT7).

- Training: identifying and problem-solving barriers to PA (ВСТ8). Problem solving strategies include using: social support (BCT29), prompts/cures such as reminders (BCT23), environmental prompts such as getting exercise clothes ready (BCT24), use of imagery (BCT34), identifying negative self-talk and replacing with positive self-talk (BCT33), establishing routine (not coded).

- Education: explanation and demonstration of strength training exercises that can be done at home (BCT21 and BCT22).

- Education about the processes of behaviour change (not coded), including stages of change, and internal and external motivation.

\section{Opportunity}

\section{Physical opportunity}

- Environmental restructuring: Provision of fitness tracker which provides summary feedback about activity.

Social opportunity

- Environmental restructuring: Exercise sessions delivered in groups.

\section{Motivation}

Reflective

- Education about: i) the general health consequences of PA and inactivity (BCT1) and ii) health consequences specific to adults with mental illness (BCT2), such as reducing mental illness symptoms, 


\section{Box 1 Continued}

countering medication side effects and preventing physical illnesses with high prevalence in this group.

Automatic

- Environmental restructuring: daily self-monitoring of PA behaviour (BCT16) using objective methods (the Garmin device) and self-report (activity log).

${ }^{*}$ Nine possible intervention functions are specified in the Behaviour Change Wheel framework: education, persuasion, incentivisation, coercion, training, restriction, environmental restructuring, modelling, enablement. ${ }^{23}$ †Behaviour change techniques have been coded as (BCT) and numbered from the CALO-RE taxonomy ${ }^{24}$; techniques not listed in this taxonomy have been specified as (not coded)

have typically focused on supervised exercise interventions. Supervised exercise interventions provide opportunity (eg, access to exercise facility and professional instruction) and motivation (eg, via first-person mastery) but may not impact psychological capability (eg, problem-solving barriers) or optimally address motivation. The application of behaviour change techniques may more directly address motivation and capability. Research suggests that, of the multitude of behaviour change techniques, ${ }^{24}$ interventions involving self-monitoring of PA combined with other self-regulatory techniques (eg, goal setting) may be more effective than interventions without these techniques. ${ }^{25}$ Self-monitoring using electronic activity monitor systems (commonly known as fitness trackers) can increase PA and assist with weight management, ${ }^{26}$ and has potential for use with clinical groups ${ }^{27}$; however, acceptability and effectiveness is yet to be established in adults with mental illness. To our knowledge, no studies have compared interventions to promote PA via supervised exercise or a combination self-monitoring and other behaviour change techniques among adults with mental illness.

To address limitations of previous research and inform practice, the aim of this study is to compare the effectiveness of two interventions designed to promote PA in adults with mental illness: a supervised exercise intervention, and a motivational intervention involving self-monitoring of PA. While both interventions may increase PA, for study purposes, the hypothesis is that the motivational intervention will have a greater impact on PA adoption than the supervised exercise intervention. Secondary aims are to evaluate acceptability and participants' experiences of the interventions and explore potential mechanisms of action using the BCW and COM-B behaviour system as a frame.

\section{METHODS AND ANALYSIS}

\section{Study design}

This is a two-arm, parallel-group, randomised controlled superiority trial of interventions designed to promote adoption and maintenance of PA among adults with mental illness.

\section{Patient and public involvement}

Participant burden of the intervention and research measures was assessed using focus group interviews and informal feedback from patients participating in two pilot rounds. Development of the research question and the intervention content was based on existing community programmes developed collaboratively with people recovering from mental health issues. These programmes that have been implemented and iteratively improved based on participant feedback since 2015. Patients will not be involved in recruitment of participants or conduct of the study. Results of this study will be disseminated to participants through presentation at consumer and community forums.

\section{Setting and participants}

Participants will be recruited from outpatient clinics of public mental health services in Brisbane, Australia (Metro North Mental Health, and Metro South Addictions and Mental Health). These services provide specialist treatment for approximately 2 million residents of a catchment encompassing inner city, suburban and regional areas; approximately 15000 patients are open to the services each year. The study will be promoted across services at clinical team meetings, and staff will be asked to refer potentially eligible clients to the research team.

Following referral, researchers will contact potential participants to provide information and screen for eligibility. Individuals will be eligible if they are a current outpatient of either mental health service, aged 18-65 years, sufficiently fluent in English to complete consent and study procedures, and willing to provide consent to study participation. Exclusion criteria are: i) receiving treatment for an eating disorder; ii) self-reporting $>300$ min of moderate-to-vigorous activity in the previous week and iii) reporting medical risk factors assessed using the Adult Pre-exercise Screening System ${ }^{28}$ without clearance for participation from a medical practitioner. Screening will involve assessment of PA using an adapted version of the Active Australia questionnaire ${ }^{29}$ which asks about time spent in walking, moderate and vigorous exercise in the previous week.

The researchers will make arrangements to meet eligible individuals who are interested in participation at the intervention venue to obtain written informed consent for study participation, and to complete the baseline assessments. The interventions will be delivered at gymnasium and community facilities of Queensland Police-Citizens Youth Welfare Association (PCYC Queensland). PCYC Queensland is an established state-wide not-for-profit organisation that offers sport and recreational activities and community development initiatives. ${ }^{30}$

\section{Randomisation}

Allocation concealment will be ensured by performing allocation after completing all baseline assessments. A researcher acting on the Data Safety Monitoring Board and not directly involved with study delivery (MB) will 
allocate participants in a 1:1 ratio using block randomisation (block size of two), using a random sequence generated at randomizer.org. Participants will be advised of allocation by telephone prior to the first group session by the researcher (JC).

\section{Intervention procedure}

The study groups will be manualised motivational (MOT) or gym exercise (GYM) interventions. Both interventions are designed to enhance capability, opportunity and motivation to do $\mathrm{PA}$, using intervention functions identified in the BCW (box 1). Interventions are 16 weeks in duration, comprising two 8-week blocks: a supervised group-based component and an 8-week unsupervised component absent of researcher contact or group sessions. There will be no restrictions from participating in other therapies or programmes outside the intervention.

Supervised components of both MOT and GYM interventions involve one $60 \mathrm{~min}$ session/week at a PCYC facility located near recruitment site, in groups of up to 10 participants. The GYM intervention will be delivered by an accredited exercise physiologist (AEP); the MOT intervention will be delivered by personnel with a tertiary qualification in a health-related field (eg, physiology, public health, nutrition). The AEP and MOT facilitators will attend group sessions for GYM and MOT interventions. Participants will be sent weekly text message reminders about the group session times, with phone follow-up if sessions are missed without explanation. Structure and content of the interventions is summarised below.

\section{Motivational intervention}

Participants will be provided Garmin Vivofit 3 devices for use over the 16-week intervention. This device provides real-time feedback about daily steps, distance walked, energy expenditure and time spent in moderate-to-vigorous activity per week ('intensity minutes'). During the initial 8 weeks, participants will be asked to:

1. Attend weekly structured group motivational sessions, comprising a 10 min discussion about progress towards goals in the previous week, 20 min discussion of PA guidelines and a health-related topic, $20 \mathrm{~min}$ 'motivational exercise' and 10 min revision of goals for the coming week.

2. Complete a daily log of step count and 'intensity minutes' as displayed on the Garmin device, and self-reported time spent active in contexts: commuting, solo aerobic exercise, community PA groups and strength training.

\section{Gym exercise intervention}

Participants will be provided 16-week gym memberships at no cost. During the initial 8 weeks, participants will be asked to:

1. Attend weekly structured group exercise sessions and attend the gym at least once unsupervised to repeat exercises from the supervised session. Sessions are based on PCYC's 'Healthy Bodies, Healthy Minds' programme (HBHM), which progressively introduces exercises to equip participants with the knowledge and confidence to develop an exercise programme based on personal abilities and preferences in consultation with an AEP. The weekly group sessions comprise a $10 \mathrm{~min}$ discussion of specified exercise topics, $20 \mathrm{~min}$ of aerobic exercise (continuous or interval training) on machine of participants' choice (treadmill, stationary bike, elliptical trainer or rowing machine) at an intensity such that talking becomes difficult (ie, 'talk test') and $30 \mathrm{~min}$ of resistance training (two sets of 1015 reps, for a variety common exercises).

2. Record variables of completed exercise sessions (weight, sets and repetitions for resistance exercises, heart rate measured using heart rate monitors on exercise machines and rate of perceived exertion achieved during aerobic exercise).

\section{Intervention consistency}

Facilitators will be required to familiarise themselves with the manual, and score $100 \%$ on a quiz about content, session structure and style of delivery as applicable for GYM and MOT interventions. Session monitoring sheets specifying content of each group session will be printed on a hardcopy A4 sheet, and facilitators will be required to mark each component completed at each session. They will also be asked to document any challenges to implementation, and responses/feedback provided by participants. Session monitoring sheets will be reviewed by the study team as a record of the content delivered consistent with the manual.

\section{Adverse event reporting}

Participants will be asked to report pain or injuries from the previous week at each session; any adverse events will be reported to the data safety monitoring board and ethics committee. Participants experiencing pain related to exercise, pre-exisitng conditions or unrelated injury may be required to discontinue the intervention until medical clearance can be obtained.

\section{Data collection}

Participant information and outcome measures will be collected as summarised in the schedule of assessments (table 1). Participants will be offered gift cards for completing assessments at each timepoint: \$A20 at baseline, $\$ \mathrm{~A} 30$ at postintervention and $\$ \mathrm{~A} 40$ at follow-up. Postintervention assessments will be administered by a researcher blind to participant allocation. All participants will be invited to complete the postintervention assessments regardless of continuation with the intervention.

\section{Participant characteristics}

Mental health clinicians (psychiatrist or case manager) will be asked to provide psychiatric diagnosis (International Statistical Classification of Diseases-10 codes obtained from hospital records) at referral. Participant baseline characteristics will be assessed using questionnaire items on health and sociodemographic information 
Table 1 Schedule of assessments

\section{Enrolment Baseline}

\section{Postallocation}

\begin{tabular}{|c|c|c|c|c|c|c|}
\hline & & \\
\hline & $-t_{3}$ & $-t_{2}$ & $-t_{1}$ & $t_{0}$ & $\mathbf{t}_{8}$ & $t_{16}$ \\
\hline \multicolumn{7}{|l|}{ Interventions } \\
\hline \multicolumn{2}{|l|}{ Motivation intervention } & & & 4 & to & $\longrightarrow$ \\
\hline \multicolumn{2}{|l|}{ Gym exercise intervention } & & & 4 & - & $\longrightarrow$ \\
\hline \multicolumn{7}{|l|}{ Intake } \\
\hline \multicolumn{2}{|l|}{ Eligibility screen } & & & & & \\
\hline \multicolumn{2}{|l|}{ Informed consent } & $x$ & & & & \\
\hline \multicolumn{2}{|l|}{ Participant characteristics } & $x$ & & & & \\
\hline \multicolumn{2}{|l|}{ Allocation } & & & $x$ & & \\
\hline \multicolumn{7}{|l|}{ Assessments } \\
\hline \multicolumn{2}{|l|}{ Accelerometry } & 4 & $\longrightarrow$ & 4 & $\rightarrow$ & \\
\hline \multicolumn{2}{|l|}{ K6 } & & $x$ & & $x$ & $x$ \\
\hline \multicolumn{2}{|l|}{ BREQ-3 } & & $x$ & & $x$ & $x$ \\
\hline \multicolumn{2}{|l|}{ SIMPAQ } & & $x$ & & $x$ & $x$ \\
\hline \multicolumn{2}{|l|}{ Stage of change } & & $x$ & & $x$ & \\
\hline \multicolumn{2}{|l|}{ Physical health measures } & & $x$ & & $x$ & \\
\hline \multicolumn{2}{|l|}{ Focus groups } & & & & & $\mathrm{X}$ \\
\hline
\end{tabular}

Recommendations for Interventional Trials (SPIRIT).

$-t_{3}$, completed on referral; $-t_{2}$, initial baseline assessment; $-t_{1}$, final baseline assessment; $t_{0}$, randomisation (week 0 ); $t_{8}$, postintervention assessments (week 8); $\mathrm{t}_{16}$, follow up assessments (week 16).

BREQ-3, Behavioural Regulation in Exercise Questionnaire; K6, Kessler-6 scale of psychological distress; SIMPAQ, Simple Physical Activity Questionnaire.

(medications, education, employment, income management, sex, gender identity, ethnicity), PA attitudes (preference for PA type and satisfaction with current PA level), current and previous use of PA self-monitoring devices (smartphone health apps, pedometers and fitness trackers). Intervention preference will also be assessed ("Given what you know about the study conditions, which one would you prefer?").

\section{Outcome measures}

Accelerometry: physical activity will be objectively measured using GENEActiv Original accelerometers (GENEActiv, Activinsights, Kimbolton, UK). GENEActiv monitors are waterproof devices, similar in appearance to a wristwatch, requiring no user input. They measure motion-related and gravitational acceleration using a triaxial microelectromechanical systems accelerometer, light exposure using a photodiode and temperature using a thermistor. The sampling frequency will be set at $10 \mathrm{~Hz}$ to extend the battery life to up to 60 days. The monitors do not provide feedback about PA, limiting the potential for reactivity. ${ }^{31}{ }^{32}$ Participants will be asked to wear monitors on their non-dominant wrist but will be offered waist or upper-arm band as alternatives if wrist-wear is not possible (eg, because of discomfort or impracticality). To assess baseline habitual PA, participants will be asked to wear GENEActiv monitors 24 hours/day for seven consecutive days prior to beginning the intervention. To assess PA adoption, participants will be asked to wear GENEActiv monitors continually during the 8-week intervention (9 weeks total monitor wear).

Behavioural Regulation in Exercise Questionnaire (BREQ-3): The BREQ-3 comprises 24 items to assess amotivation, and external, introjected, identified, integrated and intrinsic behavioural regulations. ${ }^{33}$ The BREQ-3 has high testretest reliability ( $\rho=0.78-0.84$ for regulation constructs), and has been shown to be moderately predictive of exercise participation $\left(\mathrm{R}^{2}=0.25\right)^{33}$ consistent with behavioural theory that suggests motivation influences behaviour.

Stages of change: a 5-item stages of change questionnaire based on the transtheoretical model will be used for four activities: active commuting, community activity groups, solo aerobic exercise and strength training. Participants chose one of five options: I do not do this kind of activity and I do not intend to start (precontemplation); I do not do this kind of activity but I am thinking about starting (contemplation); I occasionally do this kind of activity (preparation); I do this kind of activity regularly and started in the last 6 months (action) and I do this kind of activity regularly and have been for longer than 6 months (maintenance).

Kessler-6 scale (K6): the $\mathrm{K} 6$ is a self-administered questionnaire assessing frequency of six symptoms of distress (nervous, hopeless, restless or fidgety, so sad that nothing could cheer you up, that everything was an effort, worthless) experienced in the past month using a 5-point Likert scale. The K6 has been shown to have high internal consistency and reliability (Cronbach's $\alpha=0.89),{ }^{34}$ and total classification 
accuracy was $0.92(\mathrm{SD}=0.02)$ when discriminating cases of serious mental illness from non-cases. ${ }^{35}$

Simple Physical Activity Questionnaire (SIMPAQ): SIMPAQ is a researcher-administered self-report questionnaire assessing time spent in bed, sitting or lying down, napping during the day, walking, structured exercise and incidental activities completed in the previous week. ${ }^{36}$ Psychometrics of SIMPAQ are currently being assessed in an international validation study.

Physical health: indicators of physical health will be blood pressure measured using an automatic sphygmomanometer (Omron HEM-7302) after at least 5 min rest, waist circumference measured to the nearest $1 \mathrm{~cm}$ using a tape measure, height measured to the nearest $0.1 \mathrm{~cm}$ using a stadiometer and weight measured to the nearest $0.01 \mathrm{~kg}$ using electronic scales (Charder MS 6111). Physical capacity will be measured using the $6 \mathrm{~min}$ walk test, which is a submaximal test to assess functional capacity commonly used with adults with mental illness. ${ }^{37}$ Standardised instructions and encouragement will be provided each minute consistent with guidelines. ${ }^{38}$

\section{Process evaluation}

Feasibility and acceptability of the interventions will be examined in an embedded process evaluation. Feasibility of the interventions will be assessed by comparing intervention costs (intervention equipment, staff time) with referral and uptake rates, adherence (attendance at group sessions assessed by the researcher; attendance at unsupervised sessions assessed using self-report), completion rate and reasons for non-completion. Acceptability and potential mechanisms of action will be examined using semi-structured focus group discussions of participants' experiences with the study and interventions.

\section{Data management}

Questionnaires will be administered electronically using the online survey platform Qualtrics (Qualtrics, Provo, UT); data will be exported into SPSS V.23 (SPSS, Chicago, Illinois, USA) for analysis. GENEActiv accelerometer data will be downloaded at completion of baseline and intervention monitoring periods and analysed in Matlab 2016a (The MathWorks Natick, Massachusetts, USA). Garmin Vivofit 3 devices allow access to internet and smartphone accounts, which provide detailed feedback, social networking and other functionality; however, participants will not be given access to these accounts. Hardcopy consent forms will be stored in locked filing cabinets, and electronic data will be stored on password protected drives accessible to study investigators.

\section{Data preprocessing}

Raw GENEActiv accelerometer data will be converted into $60 \mathrm{~s}$ epochs. Data will be considered valid if the accelerometer was worn for at least $80 \%$ of waking hours ${ }^{39}$ on at least 4 days of the week including at least 1 weekend day. ${ }^{40}$ Consistent with previous research, non-wear time will be defined as $\geq 90$ min with a 20 min forward-moving
$\mathrm{SD} \leq 0.05 .{ }^{41}$ Waking hours will be defined using a validated algorithm to determine sleep periods. ${ }^{42}$ Moderate-to-vigorous activity will be defined using validated thresholds. ${ }^{43}$ Accelerometer-derived MVPA at baseline and for each week of the study period will be plotted for visual comparison. Questionnaire data will be scored consistent with the questionnaire guidelines. A relative autonomy index will be calculated from the BREQ-3 questionnaire, indicating the degree to which respondents feel self-determined.

\section{Data analysis}

\section{Hypothesis testing}

The hypothesis will be tested using multiple linear regression analyses. PA adoption will be calculated as the cumulative change in accelerometer-derived moderate-to-vigorous activity between baseline and postintervention (eg, area under curve). PA adoption will be used as the dependent variable. Independent variables will include study condition (MOT or GYM), adherence (high or low attendance) and baseline relative autonomy indices. Participant baseline characteristics will be compared between groups, and analyses adjusted for any significant differences. Missing data will be handled using multiple imputation. Analyses will be conducted using an intention-to-treat approach.

\section{Sample size}

We anticipate that a sample of 150 participants will afford the opportunity to robustly test the hypothesis, which is also considered feasible to recruit over a 3 -year period. Formal analysis of statistical power will be undertaken with a preliminary sample of 30 participants at conclusion of a pilot, and the projected sample size adjusted as appropriate.

\section{Acceptability}

Qualitative analysis will employ a framework approach, which provides a structure for coding and categorising of data. ${ }^{44}$ Both deductive and inductive logic will be used to reduce and synthesise data and develop responses to questions regarding acceptability, experience and mechanisms of action. Data coded as influencing PA participation will be analysed using the components of the COM-B model as a frame.

\section{DISCUSSION}

This study is to our knowledge the first randomised trial to compare PA behavioural outcomes for two interventions designed specifically to impact PA motivation in adults with mental illness. A pragmatic approach has been taken in the design: inclusion criteria are broad with no specific diagnostic criteria to enhance potential applicability to other mental health services. Interventions will also be delivered at community facilities, likely the most practical way to implement PA interventions given accessibility and absence of gym facilities in many mental health services. ${ }^{45} 46$ 
A strength of this study is that an objective measure of PA will be used continuously during the intervention period to assess PA adoption. Recently published protocols have outlined the intended use of an objective measure of PA for one week at each of baseline and post-intervention timepoints as a primary outcome. ${ }^{478}$ Comparing a single week of monitoring preintervention and postintervention to estimate PA change is limited because the measurement may be influenced by other life circumstances (eg, participants may be out of town, or their illness symptoms may be worse during the measurement week). Understanding how PA behaviour changes over the course of an intervention will be instructive for future studies on PA interventions for this group.

A novel aspect of this study is that the use of fitness trackers for PA self-monitoring will be evaluated for adults with mental illness. Garmin Vivofits have been chosen because of their 1-year battery life thus removing the participant burden of regular recharging, which was considered unfeasible for people experiencing chronic mental health issues. The devices provide real-time feedback about activity, and detailed feedback is available by using smartphone or internet accounts; however, this online functionality will be specifically restricted because many people with mental illness do not own a smartphone or computer with internet access, and it was considered important to standardise participant interaction with the device.

Finally, this study has a strong theoretical basis, which is lacking from most PA interventions studies with adults with mental illness. ${ }^{22}$ The study interventions use intervention functions outlined in the BCW framework to impact PA behaviour by increasing opportunity, capability and motivation as outlined in the COM-B behavioural model. Participant experiences with the interventions will be evaluated qualitatively using the COM-B model as a frame, which is important for identifying effective intervention components, and ensuring patient acceptability.

\section{Author affiliations}

${ }^{1}$ QIMR Berghofer Medical Research Institute, Herston, Queensland, Australia ${ }^{2}$ Metro South Addiction and Mental Health Service, Brisbane, Queensland, Australia ${ }^{3}$ Queensland Police-Citizens Youth Welfare Association, Brisbane, Queensland, Australia

${ }^{4}$ Queensland Centre for Mental Health Research, Brisbane, Queensland, Australia ${ }^{5}$ School of Medicine, The University of Queensland, Brisbane, Queensland, Australia ${ }^{6}$ Queensland Brain Institute, The University of Queensland, Brisbane, Queensland, Australia

${ }^{7}$ Departments of Psychiatry, Community Health \& Epidemiology, Dalhousie University, Halifax, Nova Scotia, Canada

${ }^{8}$ Metro North Mental Health Service, Brisbane, Queensland, Australia

${ }^{9}$ School of Dentistry, The University of Queensland, Brisbane, Queensland, Australia

Acknowledgements The authors would like to thank participants of pilot rounds of this study, and of the community program Healthy Bodies, Healthy Minds, for contributing to the study design by providing feedback about their experiences, preferences and perceived burden of completing research measures.

Contributors JC led the study conceptualisation, development of intervention content and writing of the protocol. SS edited the protocol. DS, SK and MB contributed to study conceptualisation. JB contributed to intervention content. SP contributed to study conceptualisation and intervention content and edited the protocol. All authors edited the manuscript.

Funding This work is supported by the Royal Brisbane and Women's Hospital (RBWH) and the RBWH Foundation, and the Study, Education and Research Trust Account (SERTA).

Competing interests The lead author is employed as a Program Manager at PCYC Queensland where he oversees implementation of the Healthy Bodies, Healthy Minds (HBHM) programme. The exercise protocol used in the HBHM programme is one of the intervention conditions of this study.

Patient consent Not required.

Ethics approval Ethics approval has been obtained from the Royal Brisbane and Women's Hospital (HREC/17/QRBW/302). The trial is registered under the Australian and New Zealand Clinical Trial Registry (ACTRN12617001017314). This manuscript is based on approved protocol V.5; any changes to protocol will be updated on the trial registry and outline in future publications. We plan to submit a manuscript on protocol development from pilot work, and a manuscript of the results to a peer-reviewed journal. Results will be presented at conferences, community and consumer forums and hospital grand rounds.

Provenance and peer review Not commissioned; externally peer reviewed.

Open access This is an open access article distributed in accordance with the Creative Commons Attribution Non Commercial (CC BY-NC 4.0) license, which permits others to distribute, remix, adapt, build upon this work non-commercially, and license their derivative works on different terms, provided the original work is properly cited, appropriate credit is given, any changes made indicated, and the use is non-commercial. See: http://creativecommons.org/licenses/by-nc/4.0/.

\section{REFERENCES}

1. Bauman AE. Updating the evidence that physical activity is good for health: an epidemiological review 2000-2003. J Sci Med Sport 2004;7:6-19.

2. Lee IM, Shiroma EJ, Lobelo F, et al. Effect of physical inactivity on major non-communicable diseases worldwide: an analysis of burden of disease and life expectancy. Lancet 2012;380:219-29.

3. US Department of Health and Human Services. Physical Activity Guidelines Advisory Committee Report, 2008.

4. Global recommendations on physical activity for health. Geneva, Switzerland: World Health Organization, 2010:58.

5. Hallal PC, Andersen LB, Bull FC, et al. Global physical activity levels: surveillance progress, pitfalls, and prospects. Lancet 2012;380:247-57.

6. Heath GW, Parra DC, Sarmiento OL, et al. Evidence-based intervention in physical activity: lessons from around the world. Lancet 2012;380:272-81.

7. Noar SM. A 10-year retrospective of research in health mass media campaigns: where do we go from here? J Health Commun 2006;11:21-42.

8. Rebar AL, Taylor A. Physical activity and mental health; it is more than just a prescription. Ment Health Phys Act 2017.

9. Vancampfort D, Stubbs B, Mitchell AJ, et al. Risk of metabolic syndrome and its components in people with schizophrenia and related psychotic disorders, bipolar disorder and major depressive disorder: a systematic review and meta-analysis. World Psychiatry 2015;14:339-47.

10. Vancampfort D, Firth J, Schuch FB, et al. Sedentary behavior and physical activity levels in people with schizophrenia, bipolar disorder and major depressive disorder: a global systematic review and metaanalysis. World Psychiatry 2017;16:308-15.

11. Schuch FB, Vancampfort D, Richards J, et al. Exercise as a treatment for depression: A meta-analysis adjusting for publication bias. $J$ Psychiatr Res 2016;77:42-51.

12. Stonerock GL, Hoffman BM, Smith PJ, et al. Exercise as Treatment for Anxiety: Systematic Review and Analysis. Ann Behav Med 2015;49:542-56.

13. Firth J, Cotter J, Elliott R, et al. A systematic review and metaanalysis of exercise interventions in schizophrenia patients. Psychol Med 2015;45:1343-61.

14. Rosenbaum S, Tiedemann A, Sherrington C, et al. Physical activity interventions for people with mental illness: a systematic review and meta-analysis. J Clin Psychiatry 2014;75:964-74.

15. Firth J, Rosenbaum S, Stubbs B, et al. Motivating factors and barriers towards exercise in severe mental illness: a systematic review and meta-analysis. Psychol Med 2016;46:2869-81. 
16. Stubbs B, Vancampfort D, Rosenbaum S, et al. Dropout from exercise randomized controlled trials among people with depression: A meta-analysis and meta regression. J Affect Disord 2016:190:457-66.

17. Firth J, Carney R, French P, et al. Long-term maintenance and effects of exercise in early psychosis. Early Interv Psychiatry 2016.

18. Stanton R, Rosenbaum S, Kalucy M, et al. A call to action: exercise as treatment for patients with mental illness. Aust J Prim Health 2015;21:120-5

19. Lederman O, Suetani S, Stanton R, et al. Embedding exercise interventions as routine mental health care: implementation strategies in residential, inpatient and community settings. Australas Psychiatry 2017;25:451-5.

20. Vancampfort D, Stubbs B, Venigalla SK, et al. Adopting and maintaining physical activity behaviours in people with severe mental illness: The importance of autonomous motivation. Prev Med 2015;81:216-20.

21. Farholm A, Sørensen M. Motivation for physical activity and exercise in severe mental illness: A systematic review of cross-sectional studies. Int J Ment Health Nurs 2016;25:116-26.

22. Farholm A, Sørensen M. Motivation for physical activity and exercise in severe mental illness: A systematic review of intervention studies. Int J Ment Health Nurs 2016;25:194-205.

23. Michie S, van Stralen MM, West R. The behaviour change wheel: a new method for characterising and designing behaviour change interventions. Implement Sci 2011;6:42.

24. Michie S, Ashford S, Sniehotta FF, et al. A refined taxonomy of behaviour change techniques to help people change their physical activity and healthy eating behaviours: the CALO-RE taxonomy. Psychol Health 2011;26:1479-98.

25. Michie S, Abraham C, Whittington C, et al. Effective techniques in healthy eating and physical activity interventions: a meta-regression. Health Psychol 2009;28:690-701.

26. Lewis ZH, Lyons EJ, Jarvis JM, et al. Using an electronic activity monitor system as an intervention modality: A systematic review. BMC Public Health 2015;15:585.

27. Cadmus-Bertram L. Using Fitness Trackers in Clinical Research: What Nurse Practitioners Need to Know. J Nurse Pract 2017; 13:34-40.

28. Norton K, Coombes J, Hobson-Powell A, et al; Adult Pre-Exercise Screening System (APSS): Exercise and Sports Science. Australia, 2012:4.

29. Australian Institute of Health and Welfare. The Active Australia Survey: A Guide and Manual for Implementation, Analysis and Reporting. Canberra: ACT, 2004.

30. PCYC Queensland website. http://www.pcyc.org.au/ (accessed 28th March 2018).

31. Clemes SA, Matchett N, Wane SL. Reactivity: an issue for short-term pedometer studies? Br J Sports Med 2008;42:68-70.
32. Clemes SA, Parker RA. Increasing our understanding of reactivity to pedometers in adults. Med Sci Sports Exerc 2009;41:674-80.

33. Wilson PM, Rodgers WM, Loitz CC, et al. "It's Who I Am ... Really!' The Importance of Integrated Regulation in Exercise Contexts1. J Appl Biobehav Res 2006;11:79-104.

34. Kessler RC, Andrews G, Colpe LJ, et al. Short screening scales to monitor population prevalences and trends in non-specific psychological distress. Psychol Med 2002;32:959-76.

35. Kessler RC, Barker PR, Colpe LJ, et al. Screening for serious mental illness in the general population. Arch Gen Psychiatry 2003;60:184.

36. Rosenbaum S, Ward PB. International Working Group. The Simple Physical Activity Questionnaire. Lancet Psychiatry 2016;3:e1.

37. Bernard P, Romain AJ, Vancampfort D, et al. Six minutes walk test for individuals with schizophrenia. Disabil Rehabil 2015;37:921-7.

38. ATS Committee on Proficiency Standards for Clinical Pulmonary Function Laboratories. ATS statement: guidelines for the six-minute walk test. Am J Respir Crit Care Med 2002;166:111.

39. Chapman JJ, Brown WJ, Burton NW. Defining a valid day of accelerometer monitoring in adults with mental illness. Ment Health Phys Act 2015;9:48-54.

40. Trost SG, Mclver KL, Pate RR. Conducting accelerometer-based activity assessments in field-based research. Med Sci Sports Exerc 2005;37(11 Suppl):S531-43.

41. Huberty J, Ehlers DK, Kurka J, et al. Feasibility of three wearable sensors for 24 hour monitoring in middle-aged women. BMC Womens Health 2015;15:55.

42. van Hees VT, Sabia S, Anderson KN, et al. A Novel, Open Access Method to Assess Sleep Duration Using a Wrist-Worn Accelerometer. PLoS One 2015;10:e0142533.

43. Dillon C, Powell C, Dowd K, et al, 2015. Criterion validity and calibration of the GENEActiv accelerometer in adults. Ambulatory Monitoring of Physical Activity and Movement (ICAMPAM). Limerick, Irelandlnternational Conference on

44. Ritchie J, Spencer L. Qualitative data analysis for applied policy research. In: Bryman A, Burgess R, eds. The qualitative researcher's companion. London: Routledge, 2002:305-29.

45. Firth J, Carney R, Elliott R, et al. Exercise as an intervention for first-episode psychosis: a feasibility study. Early Interv Psychiatry 2018;12:307-15.

46. Raine P, Truman C, Southerst A. The development of a community gym for people with mental health problems: Influences on psychological accessibility. J Ment Health 2002;11:43-53.

47. Williams J, Stubbs B, Gaughran F, et al. 'Walk This Way' - a pilot of a health coaching intervention to reduce sedentary behaviour and increase low intensity exercise in people with serious mental illness: study protocol for a randomised controlled trial. Trials 2016;17:594.

48. Hallgren M, Andersson V, Ekblom Ö, et al. Physical activity as treatment for alcohol use disorders (FitForChange): study protocol for a randomized controlled trial. Trials 2018;19:106. 